\title{
The Active Nucleus in NGC 4579
}

\section{Roberto B. Menezes ${ }^{1}$, João E. Steiner ${ }^{1}$, Tiago V. Ricci ${ }^{1}$, and Alexandre S. Oliveira ${ }^{2}$}

\author{
${ }^{1}$ Instituto de Astronomia Geofísica e Ciências Atmosféricas, Universidade de São Paulo, Rua \\ do Matão, 1226, São Paulo, SP, Brazil \\ ${ }^{2}$ IP\&D, Universidade do Vale do Paraíba, Av. Shishima Hifumi, 2911, São José dos Campos, \\ SP, Brazil
}

Keywords. galaxies: active, galaxies: individual (NGC 4579), methods: data analysis

In this work, we present an analysis of a data cube obtained with the instrument IFU/GMOS Gemini North telescope centered on the nuclear region of the LINER galaxy NGC 4579. This galaxy is known to have a type 1 AGN (see Eracleous et al. 2002 for a review). The methodology used for the analysis of the data cube was PCA tomography (Steiner et al. 2009), which consists of applying the statistical tool known as principal component analysis (PCA) to extract information from data cubes. The analysis was performed in the following way: first, the data cube for this object was transformed into a data matrix, where each row corresponded to a spatial pixel and each column corresponded to a spectral pixel. We then applied the PCA to this matrix, obtaining eigenvectors as a function of spectral pixels (which correspond to the original coordinates). These eigenvectors, which have the appearance of spectra, are called eigenspectra. We also calculated the projection of the data corresponding to the spatial pixels on each one of the eigenvectors. These images were called tomograms. Figure 1 shows Eigenspectrum E2 and tomogram T2 superposed on T1.
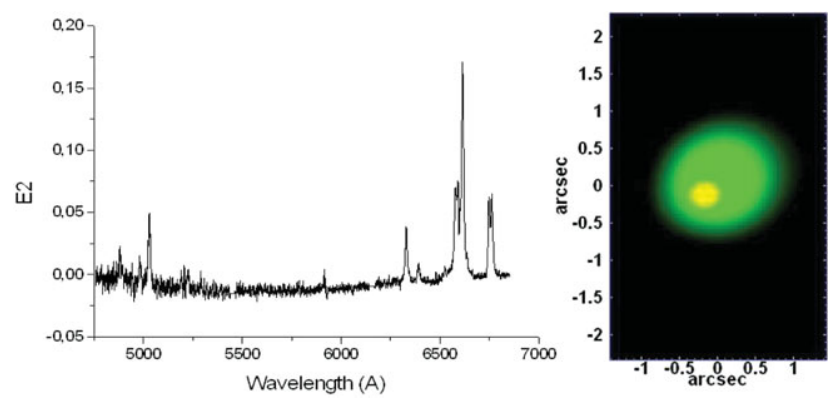

Figure 1. The eigenspectrum 2 (left), showing the type 1 AGN. Right: Tomogram 2 (AGN) superposed on tomogram 1 (bulge), showing a $0 .{ }^{\prime \prime} 3$ displacement.

Eigenvector E2 shows the presence of a type 1 AGN in this galaxy. The broad wings in $\mathrm{H} \alpha$ are clearly seen. Other lines typically present in LINERS are seen as well. Our analysis also revealed that this AGN is not located at the center of the stellar bulge, but at a displacement of $0 . " 3$ from it. This could be due to some differential reddening in the bulge or to a true geometric displacement of unknown origin.

\section{References}

Steiner, J. E., Menezes, R. B., Ricci, T. V., \& Oliveira, A. S. 2009, MNRAS, 395, 64

Eracleous, M., Shields, J. C., Chartas, G., \& Moran, E. C. 2002, ApJ, 565, 108 